\title{
Diffuse large B-cell lymphoma in colon confounded by prior history of colorectal cancer: A case report and literature review
}

\author{
YANLING REN $^{1}$, ZHILU CHEN ${ }^{2}$, CHUANYONG SU ${ }^{2}$, HONGYAN TONG $^{1}$ and WENBIN QIAN ${ }^{1}$ \\ ${ }^{1}$ Department of Hematology, The First Affiliated Hospital of Zhejiang University, College of Medicine, Zhejiang University, \\ Hangzhou, Zhejiang 310003; ' 2Department of Hematology, Tongde Hospital of Zhejiang, Hangzhou, Zhejiang 310012, \\ P.R. China
}

Received December 10, 2014; Accepted November 13, 2015

DOI: $10.3892 / 01.2016 .4078$

\begin{abstract}
A 66-year-old male underwent left hemicolectomy for rectal adenocarcinoma in 2008. Five years later he was admitted to hospital with abdominal pain. A computed tomography scan revealed notable thickening of the middle of the ascending colon wall, and colonoscopy revealed an ulcerofungating mass of $3 \times 3 \mathrm{~cm}$ in the cecum and extending to the ascending colon. Under the consideration of cancer recurrence, laparoscopic right hemicolectomy was performed directly. Surgical specimens revealed sheets of large pleomorphic lymphoid cells with nuclei of different sizes, nucleoli and mitotic phases visible in most cells. These tested positive for CD45, CD20 and CD79a diffusely, but negative for CD3, CD5, Bcl-2, Bcl-6 and ALK. The Ki-67 proliferation index was $40 \%$. Epstein-Barr virus in situ hybridization did not reveal any positive signals in any of the tumor cells. Based on these findings, the recurrent tumor was diagnosed as diffuse large B-cell lymphoma. The patient could have avoided surgery and received chemotherapy only; however, the case was confounded by the patient's prior history of colorectal cancer due to the rarity of colon lymphoma following rectal cancer in the same patient. It is therefore essential to investigate carefully and differentiate between potential lesions during routine postoperative colonoscopy following colorectal cancer surgery, as patients may present with rare colon lymphoma, which may be confused with a recurrence of colorectal cancer.
\end{abstract}

\section{Introduction}

In recent years, due to improvements in cancer diagnosis and treatment, the survival time of cancer patients has been

Correspondence to: Dr Hongyan Tong, Department of Hematology, The First Affiliated Hospital, College of Medicine, Zhejiang University, 79 Qingchun Road, Hangzhou, Zhejiang 310003, P.R. China

E-mail: yanlingrenxm@163.com

Key words: diffuse large B-cell lymphoma, colorectal cancer, rectal adenocarcinoma, second primary malignancy, rituximab prolonged considerably. However, the incidence of a second primary malignancy has also increased. In the majority of cases, the secondary cancer occurs $>6$ months after the primary cancer (1). The precise pathogenesis remains unknown; however, it may be associated with the radio- and chemotherapy used to treat the initial tumor (1). The colon is the most frequently involved organ. Ueno et al identified that colon cancer has the second highest risk of secondary malignancies (2). The New South Wales Central Cancer Registry also revealed that following rectal cancer, the risk of repeat occurrence of colon cancer, prostate and pancreas cancer, and particularly adenocarcinoma is increased, although the occurrence of malignant lymphoma following colorectal cancer surgery is rare (3). Here we report a case of primary diffuse large B-cell lymphoma (DLBCL) of the colon in a 66-year-old male, five years after he received rectal cancer surgery. Written informed consent was obtained from the patient.

\section{Case report}

A 66-year-old male was admitted to the anorectal department of The First Affiliated Hospital of Zhejiang University School of Medicine (Hangzhou, China) on October 22, 2013, due to abdominal pain for a week, and stool changes that had started a few weeks earlier, and become more noticeable recently. No family cancer history and no fever were noted. Physical examination revealed a mass in the right lower quadrant and old surgical scars, with tenderness on palpation. There was no enlargement of the lymph nodes and hepatosplenomegaly on palpation. It was noted from the patient's medical records that he had undergone partial resection of the rectum for Dukes $\mathrm{C}$ colorectal cancer (pT3N2M0, restaging by the American Joint Committee on Cancer staging system, 7th edition) in October 2008. The patient had been regularly followed up as recommended by the National Comprehensive Cancer Network practice guidelines and was considered to be free of disease from March 2012.

Under the suspicion of colon cancer recurrence, an abdominal computed tomography (CT) scan was performed. The scan revealed notable thickening in the middle of the ascending colon wall. Lumen stenosis as well as a grainy high density shadow in the fat space around the colon were observed as well as an increased lymph shadow. There was 

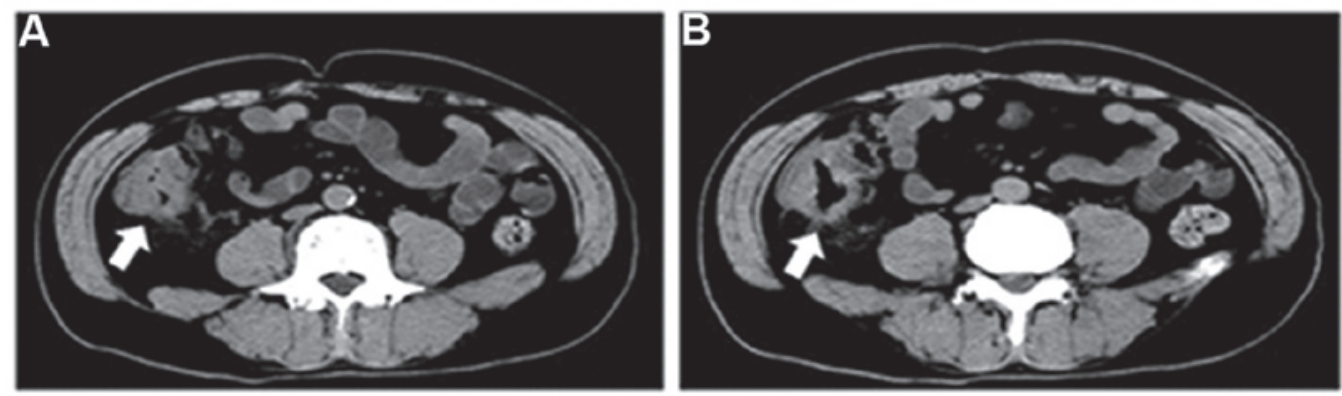

Figure 1. Computed tomography scan reveals irregular heterogeneous wall thickening in the (A) cecum and (B) ascending colon (arrowheads).
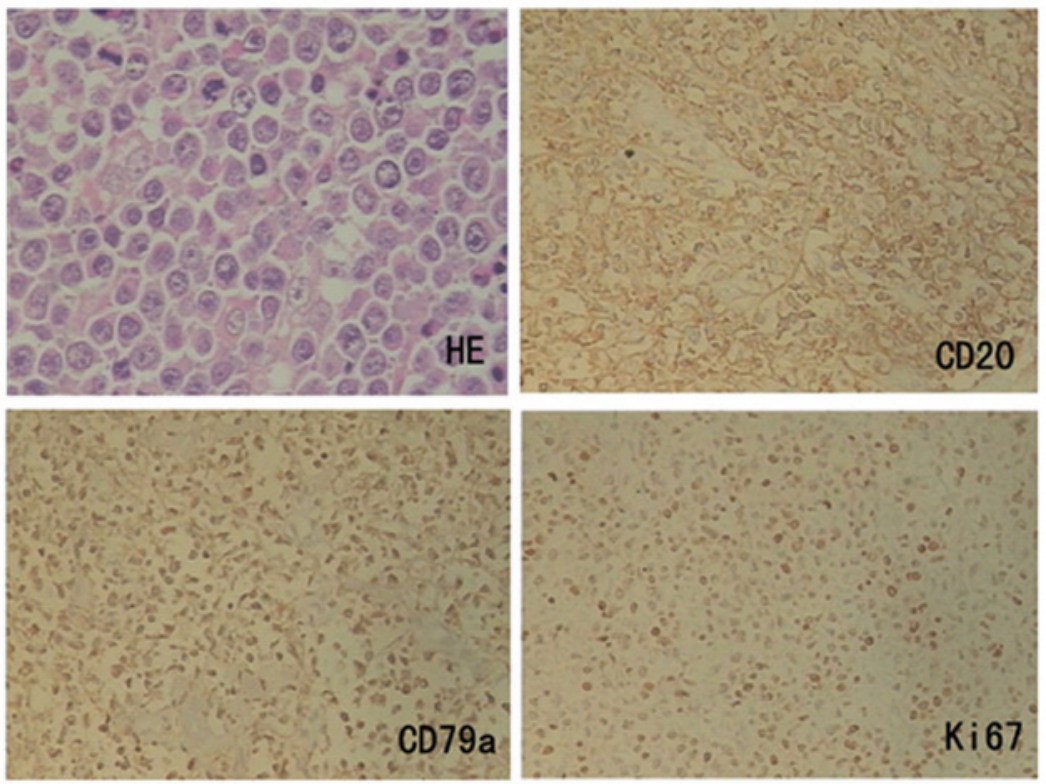

Figure 2. Sheets of large pleomorphic lymphoid cells with nuclei of different sizes, nucleoli and mitotic phases visible in most cells. Tumor cells were positively stained for CD45, CD20 and CD79a diffusely. Hematoxylin and eosin staining; magnification, x400.

no hepatomegaly, splenomegaly or metastasis (Fig. 1). While evaluating the evidence of recurrence, colonoscopy was scheduled. An ulcerofungating mass of $3 \times 3 \mathrm{~cm}$ was detected in the cecum, which extended to the ascending colon. Nonspecific inflammation was denoted in a pathology specimen taken via endoscopy. The patient underwent colon resection and enterolysis; he was discharged on the fifth postoperative day.

The specimen from the laparoscopic right hemicolectomy demonstrated an ulcerative mass, measuring $8 \times 5 \mathrm{~cm}$. Microscopic examination revealed an ulcerative tumor composed of sheets of large pleomorphic lymphoid cells with nuclei of different sizes, nucleoli and mitotic phases visible in most cells. On histochemical study, the tumor cells were positively stained for CD45, CD20 and CD79a diffusely, but negative for CD3, CD5, Bcl-2, Bcl-6 and ALK (Fig. 2). The Ki-67 proliferation index was $40 \%$ (Fig. 2), and in situ hybridization revealed negative Epstein-Barr virus, indicating DLBCL, non-germinal center B-type. Tumoral infiltration was detected in two of the 21 lymph nodes dissected from the intestinal meso.

The patient was admitted to the Department of Hematology, The First Affiliated Hospital of Zhejiang University. Hematological examination on admission revealed a white blood cell count of $7900 / \mathrm{m}^{3}$ with normal differential, a hemoglobin level of $125 \mathrm{~g} / \mathrm{l}$, and a platelet count of $204,000 / \mathrm{mm}^{3}$. The serum LDH level was 209 U/1 (normal), and bone marrow biopsy reveled no lymphoma involvement. A positron emission tomography (PET) scan was performed to stage the disease. Increased flurodeoxyglucose uptake was noted in the distal colon tissue from the anastomotic site, indicating inflammation, but a neoplasm could not be excluded. The patient was given four courses of cyclophosphamide, doxorubicin, vincristine and prednisone and rituximab (R-CHOP) chemotherapy, then the PET scan was performed again. No increased flurodeoxyglucose uptake was noted, and another two courses of R-CHOP were administered. Rituximab was administered as maintenance therapy every month. The chemotherapy course is now completed and the patient is being followed up.

\section{Discussion}

Colorectal lymphoma constitutes 6-12\% of all gastrointestinal lymphomas (4). The involvement of most colorectal lymphomas is normally secondary to widespread diseases. Primary colorectal lymphoma is extremely rare, constituting only $0.2 \%$ of all malignant tumors arising from the colorectal region, with the cecum, ascending colon and rectum most commonly 
affected (5). Our case fulfilled Dawson's criteria which was used for labeling primary gastrointestinal lymphoma, but his history of colorectal cancer made it an even rarer disease. According to the literature, there have only been three cases of malignant lymphoma following colorectal cancer. Ikeda et al reported a case of peripheral T-cell lymphoma developing at the ileocolonic anastomosis site two years after colectomy for adenocarcinoma (6), and Liao et al reported on a patient who received concurrent chemoradiotherapy for sigmoid adenocarcinoma and developed mantle cell lymphoma in the duodenal bulb 20 months later (7). Shaheen and Guddati reported a case of secondary mucosa-associated lymphoid tissue lymphoma of the colon (8). However, no cases of DLBCL following colorectal cancer have been reported until now. The etiology is unknown, but the risk factors involved may include tobacco and alcohol intake, infections and immunosuppression, genetic predisposition, and toxic effects related to treatment by chemotherapy or radiotherapy (9).

Colorectal lymphoma predominantly affects males in their $40 \mathrm{~s}$ to $60 \mathrm{~s}$, and presents as abdominal pain, loss of weight, palpable abdominal mass or lower gastrointestinal bleeding $(4,5)$. Obstruction and perforation are relatively rare in patients with colorectal lymphoma (10). Lymphoma of the colorectal region is mostly of B-cell lineage, as with other sites of the gastrointestinal tract. Endoscopically, lymphoma appears as fungating, ulcerative, infiltrative, ulcerofungating or ulcer infiltrative type, with fungating and ulcerofungating types being most common (11). The radiological appearance of colorectal lymphoma is variable and significantly overlaps with other benign and malignant conditions of the colorectal region. It was therefore difficult to differentiate it from adenocarcinoma recurrence in our case.

Treatment for colorectal lymphoma usually involves surgery and chemotherapy. Currently, due to the introduction of new active drugs as monoclonal antibodies like rituximab as part of chemotherapy treatment (12), the role of surgery is debatable. Certain authors propose that surgery may be beneficial to prevent perforation or bleeding (13), and Huang et al proposed that radical surgery could significantly increase patients' overall survival rate, compared with chemotherapy alone and palliative surgery (14). However, other authors have suggested that early diagnosis and chemotherapy might avoid a surgical procedure. Pascual et al suggested that surgery may be beneficial in patients at risk of complications including hemorrhage, obstruction and perforation, but it should be associated with postoperative chemotherapy (15). In our case, the patient underwent surgery and suffered serious postoperative diarrhea. Unfortunately, there are no controlled trials providing evidence of the optimal therapeutic approach in patients with primary colorectal lymphoma.

The survival rate of colorectal lymphoma is poor. The nonspecific symptoms make this entity difficult to diagnose and, in the majority of cases, the disease is advanced at the time of the first treatment. Histological grade and need for emergency surgery appear to be factors affecting survival.
In conclusion, lymphoma of the colon and rectum is a rare tumor. A second DLBCL following successful resection of the primary adenocarcinoma of the rectum has never been previously reported. A combination of chemotherapy and surgery appears to be the most effective treatment for the majority of patients. However, the optimal management of lymphoma of the colon and rectum has not been established. This case illustrates the need for heightened awareness of the possibility of development of secondary malignancies, particularly lymphoma, following hemicolectomy, and not only recurrence of the original cancer.

\section{References}

1. Morton LM, Swerdlow AJ, Schaapveld M, Ramadan S, Hodgson DC, Radford J and van Leeuwen FE: Current knowledge and future research directions in treatment-related second primary malignancies. EJC Suppl 12: 5-17, 2014.

2. Ueno M, Muto T, Oya M, Ota H, Azekura K and Yamaguchi T: Multiple primary cancer: an experience at the Cancer Institute Hospital with special reference to colorectal cancer. Int J Clin Oncol 8: 162-167, 2003.

3. Cooperberg MR and Fiedler PN: Ki-1 anaplastic large-cell lymphoma occurring at the site of ileocolonic anastomosis in a patient treated surgically for colonic adenocarcinoma: case report and review of the literature. Ann Diagn Pathol 5: 162-167, 2001.

4. Dionigi G, Annoni M, Rovera F, Boni L, Villa F, Castano P, Bianchi V and Dionigi R: Primary colorectal lymphomas: review of the literature. Surg Oncol (16 Suppl 1): S169-S171, 2007.

5. Stanojevic GZ, Nestorovic MD, Brankovic BR, Stojanovic MP, Jovanovic MM and Radojkovic MD: Primary colorectal lymphoma: an overview. World J Gastrointest Oncol 3: 14-18, 2011.

6. Ikeda J, Yamauchi A, Hoshida Y, Okamura S, Hashimoto K, Aozasa K and Morii E: Peripheral T-cell lymphoma developing at ileocolonic anastomosis site after colectomy for adenocarcinoma. Pathol Res Pract 206: 376-378, 2010.

7. Liao MT, Cheng MF, Chang WC, Wu YC, Lee HS and Tsai SH: Duodenal mantle cell lymphoma in a patient with advanced sigmoid adenocarcinoma. South Med J 102: 429-431, 2009.

8. Shaheen S and Guddati AK: Secondary mucosa-associated lymphoid tissue (MALT) lymphoma of the colon. Med Oncol 30: $502,2013$.

9. Cui Y, Liu T, Zhou Y, Ji Y, Hou Y, Jin W and Feng Y: Five cases report of solid tumor synchronously with hematologic malignancy. Cancer Res Treat 44: 63-68, 2012.

10. Gonzalez QH, Heslin MJ, Dávila-Cervantes A, Alvarez-Tostado J, de los Monteros AE, Shore G and Vickers SM: Primary colonic lymphoma. Am Surg 74: 214-216, 2008.

11. Myung SJ, Joo KR, Yang SK, Jung HY, Chang HS, Lee HJ, Hong WS, Kim JH, Min YI, Kim HC, et al: Clinicopathologic features of ileocolonic malignant lymphoma: analysis according to colonoscopic classification. Gastrointest Endosc 57: 343-347, 2003.

12. Salar A, Domingo-Domenech E, Estany C, Canales MA Gallardo F, Servitje O, Fraile G and Montalbán C: Combination therapy with rituximab and intravenous or oral fludarabine in the first-line, systemic treatment of patients with extranodal marginal zone B-cell lymphoma of the mucosa-associated lymphoid tissue type. Cancer 115: 5210-5217, 2009.

13. Bairey O, Ruchlemer R and Shpilberg O: Non-Hodgkin's lymphomas of the colon. Isr Med Assoc J 8: 832-835, 2006.

14. Huang S, Zheng ZX, Xu Q and Yuan XH: Diagnosis and treatment of primary colorectal non-Hodgkin's lymphoma: Analysis of 52 cases. Zhonghua Zhong Liu Za Zhi 35: 305-308, 2013 (In Chinese).

15. Pascual M, Sánchez-González B, García M, Pera M and Grande L: Primary lymphoma of the colon. Rev Esp Enferm Dig 105: 74-78, 2013. 